\title{
Closure properties of classes of multiple testing procedures
}

\author{
Georg Hahn \\ Department of Mathematics, Imperial College London
}

\begin{abstract}
Statistical discoveries are often obtained through multiple hypothesis testing. A variety of procedures exists to evaluate multiple hypotheses, for instance the ones of BenjaminiHochberg, Bonferroni, Holm or Sidak. We are particularly interested in multiple testing procedures with two desired properties: (solely) monotonic and well-behaved procedures. This article investigates to which extent the classes of (monotonic or well-behaved) multiple testing procedures, in particular the subclasses of so-called step-up and step-down procedures, are closed under basic set operations, specifically the union, intersection, difference and the complement of sets of rejected or non-rejected hypotheses. The present article proves two main results: First, taking the union or intersection of arbitrary (monotonic or well-behaved) multiple testing procedures results in new procedures which are monotonic but not well-behaved, whereas the complement or difference generally preserves neither property. Second, the two classes of (solely monotonic or well-behaved) step-up and step-down procedures are closed under taking the union or intersection, but not the complement or difference.
\end{abstract}

Keywords: Multiple Hypothesis Testing, Statistical Significance, Step Up Procedure, Set Operations, Monotonicity

\section{Introduction}

Multiple testing is a widespread tool to evaluate scientific studies (Westfall and Young, 1993 , Hsu, 1996; Hochberg and Tamhane, 2008). We are interested in testing $m \in \mathbb{N}$ hypotheses $H_{01}, \ldots, H_{0 m}$ with corresponding p-values $p_{1}, \ldots, p_{m}$ for statistical significance while controlling an error criterion such as the familywise error (FWER) or the false discovery rate (FDR). Following Gandy and Hahn (2016), we define a multiple testing procedure as a mapping

$$
h:[0,1]^{m} \times[0,1] \rightarrow \mathcal{P}(\{1, \ldots, m\})
$$

whose input is a vector of $m$ p-values $p \in[0,1]^{m}$ and a significance level $\alpha \in[0,1]$ and whose output is the set of indices of rejected hypotheses, where $\mathcal{P}$ denotes the power set.

Many procedures of the above form are available in the literature in order to correct for multiple tests, for instance the procedures of Bonferroni (1936), Sidak (1967), Holm (1979), Hochberg (1988) or Benjamini and Hochberg (1995). Many common procedures, including the ones aforementioned, belong to a certain class of procedures, called step-up and step-down procedures (Romano and Shaikh, 2006). It is assumed throughout the article that only the $m$ p-values which serve as input to $h$ are used as a basis for making decisions, dependencies between elementary hypotheses are not considered explicitly. Apart from defining properties on $p$ imposed by those multiple testing procedures to which the results of this article are applied, no additional conditions on $p$ are required. 
This article focuses on two types of multiple testing procedures: monotonic procedures defined in Roth (1999) and Tamhane and Liu (2008) as well as well-behaved procedures (Gandy and Hahn, 2016). We investigate to which extent the class of solely monotonic and the class of wellbehaved multiple testing procedures is closed under the computation of the union, intersection, difference or the complement of sets of rejected or non-rejected hypotheses.

A multiple testing procedure is said to be monotonic if smaller p-values (Tamhane and Liu, 2008) or a higher significance level (Roth, 1999) lead to more rejections. Gandy and Hahn (2016) call a monotonic multiple testing procedure well-behaved if p-values corresponding to rejected hypotheses can be lowered and p-values corresponding to non-rejected hypotheses can be increased while leaving all rejections and non-rejections invariant.

For a set of given hypotheses, the closed testing procedure (CTP) of Marcus et al. (1976) (also referred to as the closure principle) and the partitioning principle (PP) of Finner and Strassburger (2002) provide means to efficiently construct a simultaneous hypothesis test controlling the FWER. The CTP is based on enforcing coherence (Gabriel, 1969): An intersection hypothesis $H_{I}$, that is a hypothesis of the form $H_{I}=\cap_{i \in I} H_{i}$ for $I \subseteq\{1, \ldots, m\}$, is rejected if and only if all intersection hypotheses implying $H_{I}$ are rejected by their local tests (Hommel et al., 2007). Many common procedures such as the one of Holm (1979) can be constructed using the CTP. The PP divides the parameter space underlying the hypotheses of interest into disjoint subsets which are then tested independently at level $\alpha$. Since the partitioned hypotheses are disjoint, no multiplicity correction is necessary and at most one of the mutually exclusive hypotheses is true. Whereas CTP and PP can only be used to construct procedures with FWER control, the present article offers a means to combine procedures controlling several criteria such as the FDR into one procedure (see the example in Section 4.5). In case of the CTP, the exponential number of tests to be carried out might also pose a problem: The present article considers the direct construction of step-up and step-down procedures which allow for efficient testing of multiple hypotheses.

The motivation for the present article is as follows:

1. Investigating closure properties (in a set theoretical sense) of a class, in the case of the present article certain classes of multiple testing procedures, is of interest in its own right: The closure of step-up and step-down procedures allows us to construct new multiple testing procedures of the same (step-up/step-down) form from existing ones; moreover, the resulting procedure will be given explicitly.

2. Being able to perform set operations with multiple testing procedures is useful in practice: Many multiple testing procedures exist to test hypotheses according to various criteria, each of which might prove beneficial in certain applications. Whereas hypotheses can also be tested sequentially using several procedures, it is nontrivial a priori that procedures can be combined to test multiple hypotheses in a single run while drawing benefits of several criteria simultaneously. This feature is similar to using (stepwise) "shortcut procedures" (Romano and Wolf, 2005 Hommel et al., 2007) which aim to reduce the (potentially) exponential number of tests required by the CTP for FWER control to a polynomial number of tests.

3. Monotonic and well-behaved procedures have already been of interest in the literature. For instance, Gordon (2007) uses the idea of monotonicity to show that there is no monotonic stepup procedure which improves upon the Bonferroni (1936) procedure in the sense that it always returns the same rejections or possibly more. Gordon and Salzman $(2008)$ show that the classical Holm (1979) procedure dominates all monotonic step-down multiple testing procedures in the 
above sense. Proving that certain classes of procedures (for instance, monotonic procedures) are closed renders the applicability of known results more apparent.

4. The results discussed in this paper extend the methodology developed in Gandy and Hahn (2014) and Gandy and Hahn (2016) which relies on well-behaved procedures. Briefly, the authors consider a scenario in which the p-value underlying each hypothesis is unknown, but can be estimated through Monte Carlo samples drawn under the null, for instance using bootstrap or permutation tests. Instead of using estimated p-values to obtain ad-hoc decisions on all hypotheses, the authors prove that it is possible to improve existing algorithms designed for Monte Carlo based multiple testing (Besag and Clifford, 1991, Lin, 2005, van Wieringen et al., 2008, Guo and Peddada, 2008; Sandve et al., 2011): the proposed modifications guarantee that the test results of published algorithms are identical (up to an error probability pre-specified by the user) to the ones obtained with the unknown p-values. This ensures the repeatability and objectivity of multiple testing results even in the absence of p-values.

The article is structured as follows. Section 2 provides formal definitions of the two properties of a multiple testing procedure under investigation. Section 3 considers arbitrary (solely monotonic or well-behaved) multiple testing procedures and demonstrates that solely the monotonicity is preserved when taking unions and intersections. The difference and complement are neither monotonic nor well-behaved. Section 4 focuses on step-up and step-down procedures and shows that both classes of (solely monotonic or well-behaved) step-up and step-down procedures are closed under the union or intersection operation, but not the complement or difference. The article concludes with a short discussion in Section 5. All proofs are given in Appendix A. In the entire article, $|\cdot|$ and $\|\cdot\|$ denote the absolute value and the Euclidean norm, respectively, and $M:=\{1, \ldots, m\}$.

\section{Basic definitions}

Consider a step-up $\left(h^{u}\right)$ and step-down $\left(h^{d}\right)$ procedure

$$
\begin{aligned}
& h^{u}(p, \alpha)=\left\{i \in\{1, \ldots, m\}: p_{i} \leq \max \left\{p_{(j)}: p_{(j)} \leq \tau_{\alpha}(j)\right\}\right\}, \\
& h^{d}(p, \alpha)=\left\{i \in\{1, \ldots, m\}: p_{i}<\min \left\{p_{(j)}: p_{(j)}>\tau_{\alpha}(j)\right\}\right\},
\end{aligned}
$$

returning the set of indices of rejected hypotheses (Gandy and Hahn, 2016), where $p_{(1)} \leq p_{(2)} \leq$ $\cdots \leq p_{(m)}$ refers to the ordered p-values. Any procedure of the above form is fully characterised by a threshold function $\tau_{\alpha}:\{1, \ldots, m\} \rightarrow[0,1]$ returning the critical value $\tau_{\alpha}(i)$ each $p_{(i)}$ is compared to. A step-up procedure first determines the largest $j \in M$ such that the p-value $p_{(j)}$ lies below $\tau_{\alpha}(j)$ and then rejects all hypotheses having p-values up to $p_{(j)}$. Likewise, a step-down procedure non-rejects all those hypotheses with p-values larger or equal to the smallest p-value above the threshold function.

We now consider two useful properties of arbitrary multiple testing procedures. The first one, monotonicity, states that smaller p-values (Tamhane and Liu, 2008) or a higher significance level (Roth, 1999) lead to more rejections:

Definition 1. A multiple testing procedure $h$ is monotonic if $h(p, \alpha) \subseteq h\left(q, \alpha^{\prime}\right)$ for $p \geq q$ and $\alpha \leq \alpha^{\prime}$.

The monotonicity in $\alpha$ introduced by Roth (1999), also called $\alpha$-consistency (Hommel and Bretz, 2008), is a natural property desired for any testing procedure since testing at a more 
stringent significance level should never result in more rejections (Dmitrienko and Tamhane 2013).

Gandy and Hahn (2016) introduce another useful property, the class of well-behaved multiple testing procedures. Such procedures, in connection with a generic algorithm presented in Gandy and Hahn (2016), allow to use p-value estimates obtained with independent samples under the null to compute test results which are proven to be identical (up to a pre-specified error probability) to the ones obtained with the unknown p-values. A monotonic multiple testing procedure $h$ is well-behaved if it additionally satisfies the following condition.

Condition 1. 1. Let $p, q \in[0,1]^{m}$ and $\alpha \in \mathbb{R}$. If $q_{i} \leq p_{i} \forall i \in h(p, \alpha)$ and $q_{i} \geq p_{i} \forall i \notin h(p, \alpha)$, then $h(p, \alpha)=h(q, \alpha)$.

2. Fix $p^{*} \in[0,1]^{m}$ and $\alpha^{*} \in[0,1]$. Then there exists $\delta>0$ such that $p \in[0,1]^{m}, \alpha \in[0,1]$ and $\max \left(\left\|p-p^{*}\right\|,\left|\alpha-\alpha^{*}\right|\right)<\delta$ imply $h(p, \alpha)=h\left(p^{*}, \alpha^{*}\right)$.

Well-behaved procedures stay invariant if rejected (non-rejected) p-values are replaced by smaller (larger) values. Moreover, well-behaved procedures are constant on a $\delta$-neighbourhood around fixed inputs $p^{*}$ and $\alpha^{*}$.

The level $\alpha$ is a parameter in Condition 1 to account for settings in which $\alpha$ is unknown a-priori: This can occur, for instance, when the significance level depends on an estimate of the proportion of true null hypotheses which is often a functional of $p$ (Gandy and Hahn, 2016 , Section 2.2). Condition 1 is a generalisation of (Gandy and Hahn, 2014, Condition 1) which states the same invariance property for the case that $\alpha$ is a given constant: In this case, $h$ is solely a function of $p$ and the condition $\left|\alpha-\alpha^{*}\right|<\delta$ in the second part of Condition 1 can be ignored.

\section{$3 \quad$ Arbitrary multiple testing procedures}

We define the union, intersection, difference and the complement of two procedures to be the equivalent operations on the sets of rejected hypotheses returned by the two procedures. Formally, for two multiple testing procedures $h_{1}$ and $h_{2}$ we define

$$
\begin{aligned}
& h_{1} \cup h_{2}:[0,1]^{m} \times[0,1] \rightarrow \mathcal{P}(\{1, \ldots, m\}), \\
& h_{1} \cup h_{2}(p, \alpha):=h_{1}(p, \alpha) \cup h_{2}(p, \alpha),
\end{aligned}
$$

and similarly $h_{1} \cap h_{2}, h_{1} \backslash h_{2}$ and the complement $h_{i}(p, \alpha)^{c}:=\{1, \ldots, m\} \backslash h_{i}(p, \alpha)$, where $i \in\{1,2\}$.

In what follows, we sometimes drop the dependence of $h(p, \alpha)$ on $p$, on $\alpha$, or on both parameters. The following lemma summarises the results.

Lemma 1. Let $h_{1}$ and $h_{2}$ be two well-behaved multiple testing procedures.

1. $h_{1} \cup h_{2}$ and $h_{1} \cap h_{2}$ are monotonic and satisfy part 2. of Condition 1 .

2. $h_{i}(p, \alpha)^{c}$ and $h_{1} \backslash h_{2}$ are not monotonic, $i \in\{1,2\}$.

As well-behaved procedures are also monotonic, the complement or difference of two procedures is also not well-behaved.

Although by Lemma 1, both the union and the intersection are monotonic, they do not necessarily allow to lower the p-values of rejected hypotheses or to increase the p-values of non-rejected hypotheses (first part of Condition 1) as demonstrated in the following two counterexamples. 
Example 1. Let $p^{*}=(0.034,0.06,1)$ and $\alpha^{*}=0.1$. Let $h_{1}$ be the Benjamini and Hochberg (1995) step-up procedure, $h_{2}$ be the Sidak (1967) step-down procedure and $h(p, \alpha)=h_{1}(p, \alpha) \cap$ $h_{2}(p, \alpha)$. Then $h_{1}\left(p^{*}, \alpha^{*}\right)=\{1,2\}, h_{2}\left(p^{*}, \alpha^{*}\right)=\{1\}$ and thus $2,3 \notin h\left(p^{*}, \alpha^{*}\right)$. However, increasing $p^{*}$ to $q=(0.034,1,1)$ results in $h_{1}\left(q, \alpha^{*}\right)=\emptyset$ and thus $h\left(q, \alpha^{*}\right)=\emptyset \neq h\left(p^{*}, \alpha^{*}\right)$.

Example 2. Let $p^{*}$ and $\alpha^{*}$ be as in Example 1. Let $h_{1}$ be a step-up procedure which uses the same threshold function as the (step-down) Sidak (1967) correction, and likewise $h_{2}$ be a step-down procedure using the same threshold function as the (step-up) Benjamini and Hochberg (1995) procedure - using (Gandy and Hahn, 2016, Lemma 3), it is straightforward to show that both procedures are well-behaved. Let $h(p, \alpha)=h_{1}(p, \alpha) \cup h_{2}(p, \alpha)$. Then $h_{1}\left(p^{*}, \alpha^{*}\right)=\{1\}$, $h_{2}\left(p^{*}, \alpha^{*}\right)=\emptyset$ and thus $h\left(p^{*}, \alpha^{*}\right)=\{1\}$. However, decreasing $p^{*}$ to $q=(0,0.06,1)$ results in $h_{2}\left(q, \alpha^{*}\right)=\{1,2\}$ and thus $h\left(q, \alpha^{*}\right)=\{1,2\} \neq h\left(p^{*}, \alpha^{*}\right)$.

Examples 1 and 2 also demonstrate that both the union and the intersection of a well-behaved step-up and a well-behaved step-down procedure are not necessarily well-behaved any more.

Although neither the class of well-behaved multiple testing procedures of general form nor the combination of a well-behaved step-up and a well-behaved step-down procedure is closed under the four set operations aforementioned, the next section proves that this holds true for the special classes of well-behaved step-up and step-down procedures individually (when taking unions and intersections).

\section{Step-up and step-down procedures}

Gandy and Hahn (2016) show that any step-up or step-down procedure (characterised by its threshold function $\tau_{\alpha}$ ) which satisfies the following condition is well-behaved:

Condition 2. $\quad$ 1. $\tau_{\alpha}(i)$ is non-decreasing in $i$ for each fixed $\alpha$.

2. $\tau_{\alpha}(i)$ is continuous in $\alpha$ and non-decreasing in $\alpha$ for each fixed $i$.

Furthermore, Gandy and Hahn (2016) verify that a large variety of commonly used procedures satisfies Condition 2 and is hence well-behaved, among them the procedures of Bonferroni (1936), Sidak (1967), Holm (1979), Hochberg (1988) or Benjamini and Hochberg (1995).

Even though (Gandy and Hahn, 2016, Lemma 3) only prove that Condition 2 is sufficient for a procedure to be well-behaved, the condition is actually also necessary:

Lemma 2. Any well-behaved step-up or step-down procedure satisfies Condition 2.

Consider two step-up procedures $h^{u}$ and $\tilde{h}^{u}$ with threshold functions $\tau_{\alpha}^{u}$ and $\tilde{\tau}_{\alpha}^{u}$ as well as two step-down procedures $h^{d}$ and $\tilde{h}^{d}$ with threshold functions $\tau_{\alpha}^{d}$ and $\tilde{\tau}_{\alpha}^{d}$.

In the following subsections we separately investigate whether the classes of step-up (stepdown) procedures are closed under each of the four set operations (union, intersection, difference and complement). Moreover, we investigate whether the subclasses of well-behaved step-up (step-down) procedures are closed. To this end, by Lemma 2, it suffices to show that the classes of step-up (step-down) procedures satisfying Condition 2 are closed. 

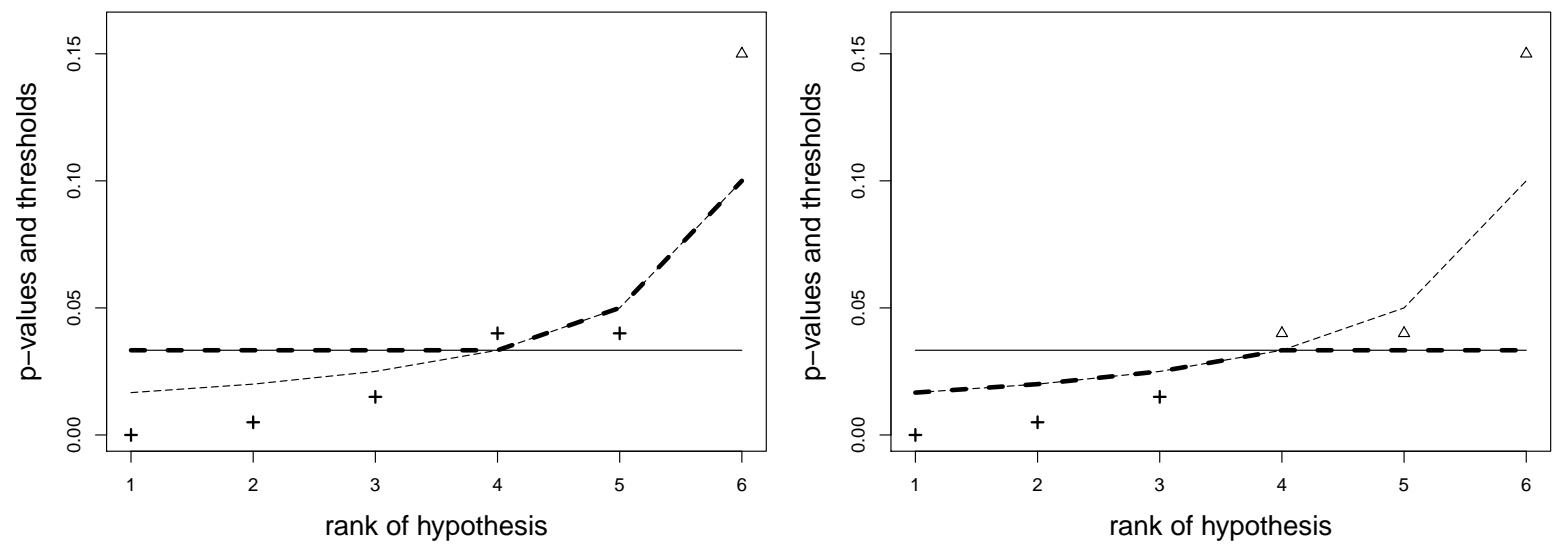

Figure 1: Combined threshold function (bold) for the computation of the union (left) and the intersection (right) of the Bonferroni (1936) correction (vertical solid line) and the Hochberg (1988) (dashed line) procedure. The Bonferroni (1936) correction was applied with significance level 0.2, the Hochberg (1988) procedure with level 0.1. P-values of rejected (crosses) and non-rejected (triangles) hypotheses.

\subsection{Union}

The class of step-up procedures is closed under the union operation: To be precise, if $h^{u}$ and $\tilde{h}^{u}$ are two step-up procedures, their union is computed by another step-up procedure $h$ with threshold function $\tau_{\alpha}(i)=\max \left(\tau_{\alpha}^{u}(i), \tilde{\tau}_{\alpha}^{u}(i)\right)$ as visualised in Fig. 1 (left).

This is seen as follows: As $\tau_{\alpha}^{u}(i), \tilde{\tau}_{\alpha}^{u}(i) \leq \tau_{\alpha}(i)$ for all $i \in M$, all hypotheses rejected by either $h^{u}$ or $\tilde{h}^{u}$ are also rejected by $h$, that is $h^{u} \cup \tilde{h}^{u} \subseteq h$. Likewise, as $\tau_{\alpha}(i)$ takes precisely one of the values $\tau_{\alpha}^{u}(i)$ or $\tilde{\tau}_{\alpha}^{u}(i)$ for each $i \in M$, any p-value belonging to the non-rejection area of both procedures $h^{u}$ and $\tilde{h}^{u}$ also stays non-rejected in $h$, hence $\left(h^{u}\right)^{c} \cap\left(\tilde{h}^{u}\right)^{c} \subseteq h^{c}$.

Moreover, the subclass of well-behaved step-up procedures is also closed under the union operation as proven in the following lemma.

Lemma 3. If $h^{u}$ and $\tilde{h}^{u}$ are two step-up procedures which satisfy Condition 2 then so does the union $h^{u} \cup \tilde{h}^{u}$.

Similarly, the union of two step-down procedures $h^{d}$ and $\tilde{h}^{d}$ (having threshold functions $\tau_{\alpha}^{d}$ and $\tilde{\tau}_{\alpha}^{d}$ ) is obtained through another step-down procedure characterised by the threshold function $\tau_{\alpha}(i)=\max \left(\tau_{\alpha}^{d}(i), \tilde{\tau}_{\alpha}^{d}(i)\right)$. Since the proof of Lemma 3 does not use any properties of $\tau_{\alpha}^{u}$ and $\tilde{\tau}_{\alpha}^{u}$ other than that both satisfy Condition 2, the maximum of two step-down threshold functions likewise leads to a threshold function satisfying Condition 2.

\subsection{Intersection}

Similarly to Section 4.1, the intersection of two step-up procedures $h^{u}$ and $\tilde{h}^{u}$ is again a step-up procedure $h$, characterised by the new threshold function $\tau_{\alpha}(i)=\min \left(\tau_{\alpha}^{u}(i), \tilde{\tau}_{\alpha}^{u}(i)\right)$ as visualised in Fig. 1 (right).

This is seen as follows: As $\tau_{\alpha}^{u}(i), \tilde{\tau}_{\alpha}^{u}(i) \geq \tau_{\alpha}(i)$ for all $i \in M$, any hypothesis non-rejected by either procedure $h^{u}$ or $\tilde{h}^{u}$ is also non-rejected by $h$, that is $\left(h^{u}\right)^{c} \cup\left(\tilde{h}^{u}\right)^{c} \subseteq h^{c}$. Likewise, as $\tau_{\alpha}(i)$ takes precisely one of the values $\tau_{\alpha}^{u}(i)$ or $\tilde{\tau}_{\alpha}^{u}(i)$ for each $i \in M$, any p-value in the rejection area of both procedures remains rejected when tested with $h$, thus $h^{u} \cap \tilde{h}^{u} \subseteq h$. 
Similarly to Lemma 3 , the subclass of well-behaved step-up procedures is again closed under the intersection operation.

Lemma 4. If $h^{u}$ and $\tilde{h}^{u}$ are two step-up procedures which satisfy Condition 2 then so does the intersection $h^{u} \cap \tilde{h}^{u}$.

The intersection of two step-down procedures $h^{d}$ and $\tilde{h}^{d}$ is again obtained with another step-down procedure using the threshold function $\tau_{\alpha}(i)=\min \left(\tau_{\alpha}^{d}(i), \tilde{\tau}_{\alpha}^{d}(i)\right)$. Analogously to Section 4.1. the proof of Lemma 4 does not use any properties of $\tau_{\alpha}^{u}$ and $\tilde{\tau}_{\alpha}^{u}$ other than that both satisfy Condition 2, thus the minimum of two step-down threshold functions again leads to a threshold function satisfying Condition 2 .

\subsection{Complement}

Whereas the complement is generally neither well-behaved nor monotonic, it can be computed for step-up and step-down procedures using the following construction.

Let $\alpha$ be a known constant. We re-consider the step-up procedure $h^{u}$ with threshold function $\tau_{\alpha}^{u}$. Then the step-down procedure $h^{d}(1-p)$ with threshold function $\tau_{\alpha}^{d}(i)=1-\tau_{\alpha}^{u}(m+1-i)$ applied to $1-p$ (instead of $p$ ) computes the complement of $h^{u}(p)$, where $1-p$ for $p \in[0,1]^{m}$ is understood coordinate-wise.

The reasoning behind this is as follows: For any hypothesis with p-value $p_{(i)}$ below $\tau_{\alpha}^{u}(i)$, $1-p_{(i)}$ (having rank $m+1-i$ in the sorted sequence of values $1-p$ ) is above $\tau_{\alpha}^{d}(m+1-i)$ by construction of $\tau_{\alpha}^{d}$. Therefore, all former rejections of $h^{u}$ turn into non-rejections of $h^{d}$ and vice versa.

Likewise, the complement of a step-down procedure $h^{d}$ with threshold function $\tau_{\alpha}^{d}$ and constant $\alpha$ is computed by a step-up procedure $h^{u}$ with threshold function $\tau_{\alpha}^{u}(i)=1-\tau_{\alpha}^{d}(m+1-i)$. Condition 2 is again satisfied:

Lemma 5. Let $\alpha$ be a known constant. If the step-up procedure $h^{u}$ with threshold function $\tau_{\alpha}^{u}$ satisfies Condition 2, then so does its step-down complement $h^{d}$ (defined with threshold function $\left.\tau_{\alpha}^{d}(i)=1-\tau_{\alpha}^{u}(m+1-i)\right)$.

The requirement that $\alpha$ be a known constant is crucial since $\tau_{\alpha}^{d}$ is not non-decreasing in $\alpha$ for a fixed $i$ as required in the second part of Condition 2. However, Lemma 5 is made possible by the fact that for a given constant $\alpha$ (that is, if $h$ and the threshold function seize to be a function of $\alpha$ ), all the parts in Condition 1 (and likewise, Condition 2 ) which involve $\alpha$ can be ignored (see remark at the end of Section 2).

\subsection{Difference}

Following the notation of Section 3 , the difference $h_{1} \backslash h_{2}$ of two procedures $h_{1}$ and $h_{2}$ can equivalently be written as $h_{1} \cap h_{2}^{c}$ using the complement of $h_{2}$. If $h_{2}$ is a step-up procedure, $h_{2}^{c}$ turns into a step-down procedure (see Section 4.3).

Therefore, in case both $h_{1}$ and $h_{2}$ are step-up (step-down) procedures satisfying Condition 2 , Lemma 1 yields that $h_{1} \backslash h_{2}$ is still monotonic but not well-behaved any more. However, if $h_{1}$ is a step-down and $h_{2}$ is a step-up procedure (or vice versa), the results from Section 4.2 apply and yield that $h_{1} \backslash h_{2}$ a well-behaved step-up/step-down procedure with explicit threshold function. 


\subsection{Example}

Suppose we are interested in testing $H_{01}, \ldots, H_{0 m}$ for statistical significance while ensuring FDR control at a pre-specified level 0.05, for instance using the Benjamini and Hochberg (1995) procedure. Additionally, we are interested in only selecting those $k \in \mathbb{N}$ hypotheses having the lowest p-values (assuming there are no ties), for instance due to the fact that budget constraints only allow follow-up studies for $k$ hypotheses. We thus look to construct an intersection procedure which returns the indices of hypotheses satisfying both requirements simultaneously.

To this end, let $h^{1}$ be the Benjamini and Hochberg (1995) step-up procedure controlling the FDR at level 0.05, defined through the threshold function $\tau^{1}(i)=0.05 \cdot i / m$ for $i \in\{1, \ldots, m\}$. Moreover, let $h^{2}$ be the (step-up) Bonferroni (1936) correction with constant but $p$-dependant threshold function $\tau_{p}^{2}(i)=p_{(k)}$ for $i \in\{1, \ldots, m\}$, where $p_{(k)}$ denotes the $k$ 'th smallest entry of vector $p=\left(p_{1}, \ldots, p_{m}\right)$. By construction, all rejected hypotheses by $h^{2}$ are precisely the ones with the $k$ lowest p-values. Threshold functions $\tau_{\alpha}$ for which $\alpha=\alpha(p)$ is a function of $p$ are widely used in practice, for instance when using an estimate of the proportion of true null hypotheses to correct the level $\alpha$ (see, for instance, Example 1 in Gandy and Hahn (2016)). Both the Benjamini and Hochberg (1995) procedure $h^{1}$ and the Bonferroni (1936) correction $h^{2}$ satisfy Condition 2 and are thus well-behaved.

Following Section 4.2 , the step-up procedure $h$ defined through the threshold function $\tau_{p}(i)=$ $\min \left(\tau^{1}(i), \tau_{p}^{2}(i)\right)=\min \left(0.05 \cdot i / m, p_{(k)}\right)$ computes $h^{1} \cap h^{2}$. Moreover, $h$ is well-behaved by Lemma 4 .

Consider the numerical example of 15 ordered p-values (here denoted as $\tilde{p}$ ) given in Section 3.2 of Benjamini and Hochberg (1995). In agreement with Benjamini and Hochberg (1995), who test $\tilde{p}$ while controlling the FDR at level 0.05 and observe four rejections (of the first four hypotheses), $h^{1}$ applied to $\tilde{p}$ yields $h^{1}(\tilde{p})=\{1,2,3,4\}$. Applying the intersection procedure $h$ constructed above with $k=3$ to $\tilde{p}$ yields $h(\tilde{p})=\{1,2,3\}$, that is $h$ indeed yields those $k=3$ hypotheses having the lowest p-values which are also significant under FDR control at level 0.05.

\section{Discussion}

This article investigates closure properties of general multiple testing procedures, step-up and step-down procedures as well as subclasses of (solely) monotonic and well-behaved procedures under four set operations (union, intersection, complement and difference).

The article shows that for general multiple testing procedures, solely the class of monotonic procedures is closed under taking the union and intersection. However, the subclass of wellbehaved step-up (step-down) procedures is closed under taking the union and intersection.

The implications of the closure properties proven in this article are threefold: They provide a tool to construct new procedures of known form and with known properties, they render theoretical results (Gordon, 2007; Gordon and Salzman, 2008) instantly applicable to a large class of multiple testing procedures and they allow to combine the benefits of various multiple testing procedures in practice.

\section{A Proofs}

The appendix contains all proofs sorted by section. 


\section{A.1 Proofs of Section 3}

Proof of Lemma 1. We prove both assertions.

1. Monotonicity. If $p \leq q$ and $\alpha \leq \alpha^{\prime}$ then $h_{1}(q, \alpha) \subseteq h_{1}\left(p, \alpha^{\prime}\right), h_{2}(q, \alpha) \subseteq h_{2}\left(p, \alpha^{\prime}\right)$ and thus $h_{1}(q, \alpha) \cup h_{2}(q, \alpha) \subseteq h_{1}\left(p, \alpha^{\prime}\right) \cup h_{2}\left(p, \alpha^{\prime}\right)$ as well as $h_{1}(q, \alpha) \cap h_{2}(q, \alpha) \subseteq h_{1}\left(p, \alpha^{\prime}\right) \cap h_{2}\left(p, \alpha^{\prime}\right)$.

The second statement of Condition 1 1 . As $h_{1}$ satisfies Condition 1 there exists $\delta_{1}$ such that $\max \left(\left\|p-p^{*}\right\|,\left|\alpha-\alpha^{*}\right|\right)<\delta_{1}$ implies $h_{1}(p, \alpha)=h_{1}\left(p^{*}, \alpha^{*}\right)$. Likewise for $h_{2}$ with a suitable $\delta_{2}$. For $\delta=\min \left(\delta_{1}, \delta_{2}\right)$ and $\max \left(\left\|p-p^{*}\right\|,\left|\alpha-\alpha^{*}\right|\right)<\delta$, we have $h_{1}(p, \alpha)=h_{1}\left(p^{*}\right.$, $\left.\alpha^{*}\right)$ and $h_{2}(p, \alpha)=h_{2}\left(p^{*}, \alpha^{*}\right)$ and thus $h_{1} \cup h_{2}(p, \alpha)=h_{1} \cup h_{2}\left(p^{*}, \alpha^{*}\right)$. Likewise for the intersection.

2. Fix $\alpha$. If $q \leq p$ then $h_{i}(p, \alpha) \subseteq h_{i}(q, \alpha)$, but $h_{i}(p, \alpha)^{c} \supseteq h_{i}(q, \alpha)^{c}$ for $i \in\{1,2\}$. The complement is thus not monotonic. The operation $h_{1}(p, \alpha) \backslash h_{2}(p, \alpha)$ is equivalent to $h_{1}(p, \alpha) \cap$ $\left(h_{2}(p, \alpha)\right)^{c}$ and thus also not monotonic.

\section{A.2 Proofs of Section 4}

Proof of Lemma 2. Let $h$ be a step-up (step-down) procedure characterised through its threshold function $\tau_{\alpha}$. We now verify Condition 2 .

1. We show that $\tau_{\alpha}(i)$ must be non-decreasing in $i$ for a fixed $\alpha$. Indeed, suppose $\tau_{\alpha}$ is decreasing for some $i$. Then $h$ cannot be monotonic for all inputs: Assume that $m=2$, $p=(0.5,0.5)$ and $h$ is of step-up type with $\tau_{\alpha}(1)=1$ and $\tau_{\alpha}(2)=0$. Then $h(p)=\{1\}$ but increasing $p$ to $q=(1,0.5)$ results in $h(q)=\{2\} \nsubseteq \subseteq h(p)$, thus contradicting monotonicity.

2. We show that $\tau_{\alpha}(i)$ must also be non-decreasing in $\alpha$ for any fixed $i$. Indeed, for a fixed $i$, suppose $\tau_{\alpha}(i)>\tau_{\alpha^{\prime}}(i)$ for $\alpha<\alpha^{\prime}$. Then $h$ can again not be monotonic for all inputs: Assume we test $m=1$ hypothesis $H_{01}$ with p-value $p=\tau_{\alpha}(1)>\tau_{\alpha^{\prime}}(1)$. Then $H_{01}$ is rejected at $\tau_{\alpha}(1)$ but non-rejected at $\tau_{\alpha^{\prime}}(1)$ even though $\alpha<\alpha^{\prime}$, thus contradicting monotonicity.

3. We show that $\tau_{\alpha}(i)$ is continuous in $\alpha$ for a fixed $i$. Let $\epsilon>0$ be given. Fix $i$ and $\alpha^{*}$. We show continuity of the threshold function at $\alpha^{*}$ as $\alpha \rightarrow \alpha^{*}$.

Case 1: $\alpha^{*}>\alpha$. Then $\tau_{\alpha^{*}}(i) \geq \tau_{\alpha}(i)$ by monotonicity. Define $p^{*}=\left(0, \ldots, 0, p_{i}^{*}, 1, \ldots, 1\right)$ for any $p_{i}^{*} \in\left[0, \tau_{\alpha^{*}}(i)\right)$ (i.e., $p^{*}$ contains $p_{i}^{*}$ as $i$ th entry, zeros before and ones after). Since $h$ is wellbehaved it satisfies the second part of Condition 1 , hence for the fixed $p^{*}$ and $\alpha^{*}$ there exists $\delta>0$ such that for all $\alpha$ and $p$ satisfying $\left|\alpha-\alpha^{*}\right|<\delta,\left\|p-p^{*}\right\|<\delta$ we have $h(p, \alpha)=h\left(p^{*}, \alpha^{*}\right)$. Assume $\left|\alpha-\alpha^{*}\right|<\delta$. Define $p=\left(0, \ldots, 0, p_{i}^{*}-\gamma, 1, \ldots, 1\right)$ for any $0<\gamma<\min (\delta, \epsilon)$. Since $\left|\alpha-\alpha^{*}\right|<\delta$ and $\left\|p-p^{*}\right\|=\gamma<\delta, h(p, \alpha)=h\left(p^{*}, \alpha^{*}\right)$ by Condition 1: As the $i$ th hypothesis is rejected in $h\left(p^{*}, \alpha^{*}\right)$ and hence also in $h(p, \alpha)$, it follows that $\tau_{\alpha^{*}}(i) \geq \tau_{\alpha}(i) \geq p_{i}=p_{i}^{*}-\gamma$. This holds true for all $p_{i}^{*} \in\left[0, \tau_{\alpha^{*}}(i)\right)$, thus $\tau_{\alpha^{*}}(i) \geq \tau_{\alpha}(i) \geq \tau_{\alpha^{*}}(i)-\gamma$ and hence $\left|\tau_{\alpha^{*}}(i)-\tau_{\alpha}(i)\right| \leq \gamma<\epsilon$.

Case 2: $\alpha^{*} \leq \alpha$. Then $\tau_{\alpha^{*}}(i) \leq \tau_{\alpha}(i)$. Using $p^{*}=\left(0, \ldots, 0, p_{i}^{*}, 1, \ldots, 1\right)$ with $p_{i}^{*} \in\left(\tau_{\alpha^{*}}(i), 1\right]$ and $p=\left(0, \ldots, 0, p_{i}^{*}+\gamma, 1, \ldots, 1\right)$ with $0<\gamma<\min (\delta, \epsilon)$, the same argument as in Case 1 yields $\tau_{\alpha^{*}}(i) \leq \tau_{\alpha}(i)<\tau_{\alpha^{*}}(i)+\gamma$

Proof of Lemma 3. Let $h=h^{u} \cup \tilde{h}^{u}$ be defined through $\tau_{\alpha}(i)=\max \left(\tau_{\alpha}^{u}(i), \tilde{\tau}_{\alpha}^{u}(i)\right)$. First, $h$ is monotonic by Lemma 1. We now verify Condition 2. 
1. The function $\tau_{\alpha}(i)$ is non-decreasing in $i$ : Suppose w.l.o.g. $\tau_{\alpha}(i)=\tau_{\alpha}^{u}(i)$. If $\tau_{\alpha}^{u}(i+1) \geq$ $\tilde{\tau}_{\alpha}^{u}(i+1)$ then $\tau_{\alpha}(i)=\tau_{\alpha}^{u}(i) \leq \tau_{\alpha}^{u}(i+1)=\tau_{\alpha}(i+1)$ by definition of $\tau_{\alpha}$ as the maximum of $\tau_{\alpha}^{u}$ and $\tilde{\tau}_{\alpha}^{u}$. If $\tau_{\alpha}^{u}(i+1)<\tilde{\tau}_{\alpha}^{u}(i+1)$ then $\tau_{\alpha}(i)=\tau_{\alpha}^{u}(i) \leq \tau_{\alpha}^{u}(i+1)<\tilde{\tau}_{\alpha}^{u}(i+1)=\tau_{\alpha}(i+1)$.

2. $\tau_{\alpha}$ is continuous in $\alpha$ as the maximum of two continuous functions (in this case in $\alpha$ ) is continuous. The function $\tau_{\alpha}$ is also non-decreasing in $\alpha$ : Indeed, fix $i$, let $\alpha \leq \alpha^{\prime}$ and suppose w.l.o.g. $\tau_{\alpha}(i)=\tau_{\alpha}^{u}(i)$. If $\tau_{\alpha^{\prime}}^{u}(i) \leq \tilde{\tau}_{\alpha^{\prime}}^{u}(i)$ then $\tau_{\alpha}(i)=\tau_{\alpha}^{u}(i) \leq \tau_{\alpha^{\prime}}^{u}(i) \leq \tilde{\tau}_{\alpha^{\prime}}^{u}(i)=\tau_{\alpha^{\prime}}(i)$ by definition of $\tau_{\alpha}$ as the maximum of $\tau_{\alpha}^{u}$ and $\tilde{\tau}_{\alpha}^{u}$. Otherwise, $\tau_{\alpha}(i)=\tau_{\alpha}^{u}(i) \leq \tau_{\alpha^{\prime}}^{u}(i)=\tau_{\alpha^{\prime}}(i)$.

Proof of Lemma 4. Let $h=h^{u} \cap \tilde{h}^{u}$ be defined through $\tau_{\alpha}(i)=\min \left(\tau_{\alpha}^{u}(i), \tilde{\tau}_{\alpha}^{u}(i)\right)$. Again, $h$ is monotonic by Lemma 1. We now verify Condition 2.

1. The function $\tau_{\alpha}(i)$ is non-decreasing in $i$ : Suppose w.l.o.g. $\tau_{\alpha}(i)=\tau_{\alpha}^{u}(i)$. If $\tau_{\alpha}^{u}(i+1) \geq$ $\tilde{\tau}_{\alpha}^{u}(i+1)$ then $\tau_{\alpha}(i)=\tau_{\alpha}^{u}(i) \leq \tilde{\tau}_{\alpha}^{u}(i) \leq \tilde{\tau}_{\alpha}^{u}(i+1)=\tau_{\alpha}(i+1)$ by definition of $\tau_{\alpha}$ as the minimum of $\tau_{\alpha}^{u}$ and $\tilde{\tau}_{\alpha}^{u}$. If $\tau_{\alpha}^{u}(i+1)<\tilde{\tau}_{\alpha}^{u}(i+1)$ then $\tau_{\alpha}(i)=\tau_{\alpha}^{u}(i) \leq \tau_{\alpha}^{u}(i+1)=\tau_{\alpha}(i+1)$.

2. $\tau_{\alpha}$ is continuous in $\alpha$ as the minimum of two continuous functions (in this case in $\alpha$ ) is continuous. The function $\tau_{\alpha}$ is also non-decreasing in $\alpha$ : Indeed, fix $i$, let $\alpha \leq \alpha^{\prime}$ and suppose w.l.o.g. $\tau_{\alpha}(i)=\tau_{\alpha}^{u}(i)$. If $\tau_{\alpha^{\prime}}^{u}(i) \leq \tilde{\tau}_{\alpha^{\prime}}^{u}(i)$ then $\tau_{\alpha}(i)=\tau_{\alpha}^{u}(i) \leq \tau_{\alpha^{\prime}}^{u}(i)=\tau_{\alpha^{\prime}}(i)$. Otherwise, $\tau_{\alpha}(i)=\tau_{\alpha}^{u}(i) \leq \tilde{\tau}_{\alpha}^{u}(i) \leq \tilde{\tau}_{\alpha^{\prime}}^{u}(i)=\tau_{\alpha^{\prime}}(i)$ (by definition of $\tau_{\alpha}$ as the minimum).

Proof of Lemma 5. Since $\tau_{\alpha}^{u}(i)$ is non-decreasing in $i$, it is immediate to verify that $\tau_{\alpha}^{d}(i)$ is also non-decreasing in $i$. For a given constant $\alpha$, the second part of Condition 2 can be ignored as shown in (Gandy and Hahn, 2014, Condition 1) and is hence automatically satisfied (see Section 2p.

\section{References}

Benjamini, Y. and Hochberg, Y. (1995). Controlling the false discovery rate: A practical and powerful approach to multiple testing. J Roy Stat Soc B Met, 57(1):289-300.

Besag, J. and Clifford, P. (1991). Sequential Monte Carlo p-values. Biometrika, 78(2):301-304.

Bonferroni, C. (1936). Teoria statistica delle classi e calcolo delle probabilità. Pubblicazioni del $R$ Istituto Superiore di Scienze Economiche e Commerciali di Firenze, 8:3-62.

Dmitrienko, A. and Tamhane, A. (2013). General theory of mixture procedures for gatekeeping. Biom J, 55(3):402-419.

Finner, H. and Strassburger, K. (2002). The partitioning principle: a powerful tool in multiple decision theory. Ann Stat, 30(4):1194-1213.

Gabriel, K. (1969). Simultaneous Test Procedures - Some Theory of Multiple Comparisons. Ann Math Statist, 40(1):224-250.

Gandy, A. and Hahn, G. (2014). MMCTest - A Safe Algorithm for Implementing Multiple Monte Carlo Tests. Scand J Stat, 41(4):1083-1101. 
Gandy, A. and Hahn, G. (2016). A framework for Monte Carlo based Multiple Testing. Scand J Stat, 43(4):1046-1063.

Gordon, A. (2007). Unimprovability of the Bonferroni procedure in the class of general step-up multiple testing procedures. Stat Probab Lett, 77(2):117-122.

Gordon, A. and Salzman, P. (2008). Optimality of the Holm procedure among general step-down multiple testing procedures. Stat Probab Lett, 78(13):1878-1884.

Guo, W. and Peddada, S. (2008). Adaptive choice of the number of bootstrap samples in large scale multiple testing. Stat Appl Genet Mol Biol, 7(1):1-16.

Hochberg, Y. (1988). A sharper Bonferroni procedure for multiple tests of significance. Biometrika, 75(4):800-802.

Hochberg, Y. and Tamhane, A. (2008). Multiple Comparison Procedures. Wiley.

Holm, S. (1979). A simple sequentially rejective multiple test procedure. Scand J Stat, 6(2):6570.

Hommel, G. and Bretz, F. (2008). Aesthetics and power considerations in multiple testing - a contradiction? Biom J, 50(5):657-666.

Hommel, G., Bretz, F., and Maurer, W. (2007). Powerful short-cuts for multiple testing procedures with special reference to gatekeeping strategies. Stat Med, 26(22):4063-4073.

Hsu, J. (1996). Multiple Comparisons: Theory and Methods. Chapman and Hall/CRC.

Lin, D. (2005). An efficient Monte Carlo approach to assessing statistical significance in genomic studies. Bioinformatics, 21(6):781-787.

Marcus, R., Peritz, E., and Gabriel, K. (1976). On closed testing procedures with special reference to ordered analysis of variance. Biometrika, 63(3):655-660.

Romano, J. and Shaikh, A. (2006). Stepup procedures for control of generalizations of the familywise error rate. Ann Stat, 34(4):1850-1873.

Romano, J. and Wolf, M. (2005). Exact and Approximate Stepdown Methods for Multiple Hypothesis Testing. J Am Stat Assoc, 100(469):94-108.

Roth, A. (1999). Multiple comparison procedures for discrete test statistics. J Stat Plan Infer, 82(1-2):101-117.

Sandve, G., Ferkingstad, E., and Nygard, S. (2011). Sequential Monte Carlo multiple testing. Bioinformatics, 27(23):3235-3241.

Sidak, Z. (1967). Rectangular confidence regions for the means of multivariate normal distributions. J Am Stat Assoc, 62(318):626-633.

Tamhane, A. and Liu, L. (2008). On weighted Hochberg procedures. Biometrika, 95(2):279-294.

van Wieringen, W., van de Wiel, M., and van der Vaart, A. (2008). A test for partial differential expression. J Am Stat Assoc, 103(483):1039-1049.

Westfall, P. and Young, S. (1993). Resampling-based multiple testing: Examples and methods for p-value adjustment. Wiley. 